\title{
Physiopathological Hypothesis of Cellulite
}

\author{
José Maria Pereira de Godoy ${ }^{1, *}$ and Maria de Fátima Guerreiro de Godoy ${ }^{2}$ \\ ${ }^{I}$ Department of Cardiology and Cardiovascular Surgery Medical School of São Jose do Rio Preto-FAMERP-Brazil \\ ${ }^{2}$ FAMERP and Clinic Godoy, São José do Rio Preto, Brazil
}

\begin{abstract}
A series of questions are asked concerning this condition including as regards to its name, the consensus about the histopathological findings, physiological hypothesis and treatment of the disease. We established a hypothesis for cellulite and confirmed that the clinical response is compatible with this hypothesis. Hence this novel approach brings a modern physiological concept with physiopathologic basis and clinical proof of the hypothesis. We emphasize that the choice of patient, correct diagnosis of cellulite and the technique employed are fundamental to success.
\end{abstract}

Key Words: Physiopathological hypothesis, cellulite.

\section{TO THE EDITOR}

Since its description, cellulite remains one of the great challenges of medicine [1,2]. A series of questions are asked concerning this condition including as regards to its name, the consensus about the histopathological findings, physiological hypothesis and treatment of the disease.

However, 10 years ago, because of clinical observations and reports from patients, our interest in this disease was aroused due to the improvement of cellulite utilizing a novel technique of lymph drainage in the treatment of lymphedema. Consequently, we started to use this therapeutic technique in patients with cellulite and found that it is possible to reduce cellulite completely. We analyzed failures in the treatment of some patients and the reasons for these failures and searched for solutions to these problems. Consequently we found it is possible to select patients and obtain success in practically $100 \%$ of cases.

Several treatment regimens were evaluated; we found that it is possible to reduce the greatest abdomen or thigh perimetric variation by $10 \mathrm{~cm}$ utilizing intensive treatment for 4 hours daily over a period of 10 days. Even with these significant reductions, weight loss does not occur. The maintenance of the results can last for at least eight years as long as the patient is not overweight or suffer from edema.

In light of these results, we established a hypothesis for cellulite and confirmed that the clinical response is compatible with this hypothesis. In a review of published histological data, we observed that the onset of changes leading to cellulite is characterized by an accumulation of fluids in the cell interstice $[1,3,4]$ and that the progression to subsequent phases suggests an increase in the concentration of substances in this space, local reactions of these substances and an increased difficultly in the exchange of particles

*Address correspondence to this author at the Rua Floriano Peixoto, 2950. São José do Rio Preto, SP - Brazil; Zipe code: 15010-020;

E-mail: godoyjmp@riopreto.com.br between the cell and the interstice. On analyzing the physiology of the mobilization of substances in the cell interstice, we identified, in terms of drainage, the involvement of both the venous and lymphatic systems. If cellulite were exclusively a problem of the venous system, the use of diuretics would be sufficient to treat or minimize the disease. The data demonstrate an accumulation of several substances $[3,4]$ that are destined to the lymph drainage system. Published data suggest dilation of the lymphatic vessels [3], however in this hypothesis we suggest there is an alteration of the lymphatic system which results in the accumulation of substances in the cell interstice. The clinical grade of the disease is related to the concentration of these substances and reactions resulting from this accumulation. Hence, we identified the mechanism involved, that is, interference in the lymphatic system.

On analyzing the lymphatic system, we observed that there is no evidence of obstruction associated to cellulite and so only interference in the contraction of the lymphangions or lymphostasis, similar to venostasis, remain as possible hypotheses. Thus, it is necessary to identify which hypothesis best explains this mechanism.

As cellulite is a disease that almost exclusively affects women, the hormonal factor draws our attention. The distribution of cellulite in woman occurs in specific regions and follows the same pattern as the deposit of adipose tissue. Hormones can stimulate the accumulation of some substances in the cell interstice similar to what occurs in cellulite, thus there seems to be a very strong link between these aspects.

Hence, there is evidence that establishes a physiopathological hypothesis of cellulite which involves the affect of female hormones in the contraction and tonus of the lymphatic vessels, with consequent lymphostasis and the accumulation of substances in the cell interstice. This accumulation may have an effect on the exchange of substances between the cell and the cell interstice and reflect 
in local reactions triggering edema and fibrosis. The total reduction of cellulite attained utilizing the lymph drainage technique supports this hypothesis. Although cellulite can be associated with obesity it also occurs in very slim women and so in our studies we excluded overweight and obese patients to characterize cellulite in isolation.

Great variations in volumes occur without weight loss leading us to speculate why this happens. The treatment employed transfers the accumulated substances into the blood flow which we believe are then deposited in other regions of the body. The improvement in the turgor of the skin after treatment suggests that these substances are deposited in the skin and subcutaneous tissue. This theory is also supported by the fact that patients with history of excessively dry skin observed an improvement. Thus, all the changes observed during treatment can be explained.

This technique has been consistently demonstrated to students who want to know and evaluate the treatment. We emphasize that the choice of patient, correct diagnosis of cellulite and the technique employed are fundamental to success. We have noticed that many students want to use this technique to treat obese individuals but they do not achieve positive results as the treatment is incorrect for obesity: a low-calorie diet may be successful. Another aspect is the presence of edema, in particular idiopathic cyclic edema. In these cases failure should be expected as the cause of the edema should be treated first [5]. When edema, obesity and cellulite (regional cutaneous lymphostasis) are associated, we suggest that the edema should first be controlled otherwise the success of treatment is threatened [2]. Thus, success demands correct diagnosis and correct treatment.

The therapeutic techniques employed in this case were developed based on scientific principles with the possibility of reproducing all the phases both in vitro and in vivo [6-10]. Several forms of treatment are suggested in the literature, but failure resides in not identifying a physiological basis that supports their application. Good results reported in publications are questionable as the treatment utilized causes cell lesions (adipocytes and other structures) due to this lack of understanding of the condition's physiological basis. The result of these treatments, even when a reduction in volume is observed, is an inflammatory process and fibrosis. The consequences are a reduction that frequently leads to flaccidity of the skin. Thus, it is necessary to re-consider these therapeutic techniques in order to find a physiological foundation for their use. It is impossible to establish the correct treatment without having a physiological understanding of the disease.

Any treatment should be non-invasive or minimally invasive in an attempt to reproduce the physiology of the organism. Hence this novel approach brings a modern physiological concept with physiopathologic basis and clinical proof of the hypothesis.

\section{REFERENCES}

[1] Alquier L. Ce qu'est la cellulite. Comment la traiter. Monde Méd 1949; 59(960): 344-9.

[2] Godoy JMP, Godoy MFG. Celulite do diagnóstico ao Tratamento. São José do Rio Preto: Talkclub 2003; p. 64.

[3] Segers AM, Abulafia J, Kriner J, Cortodono O. Celulitis: estúdio histopatológico e histoquímico de 100 casos. Med Cuton Ibero Lat Am 1984; 12(2): 167-172.

[4] Curri SB. Las paniculopatias de estasis venosa: diagnostico clinico e instrumental. Barcelona: Hausmann 1991; p. 211.

[5] Pereira de Godoy JM. Aminaphtone in idiopathic cyclic edema syndrome. Phlebology 2008; 23: 118-9.

[6] de Godoy JM, Godoy Mde F. Development and evaluation of a new apparatus for lymph drainage: preliminary results. Lymphology 2004; 37: 62-4.

[7] de Godoy JM, Batigalia F, Godoy Mde F. Preliminary evaluation of a new, more simplified physiotherapy technique for lymphatic drainage. Lymphology 2002; 35: 91-3.

[8] Pereira de Godoy JM, Godoy Mde F. Manual lymph drainage: a new concept. J Vasc Br 2004; 03: 77-80.

[9] de Godoy JM, Godoy Mde F, Meza MC. Godoy \& Godoy technique of cervical stimulation in the reduction of edema of the face after cancer treatment. QJM 2008; 101: 325-6.

[10] Pereira de Godoy JM, Silva SH, Toninato MC, Godoy Mde F. Cervical stimulation for volumetric reduction of limbs in the treatment of lymphedema. Indian J Med Sci 2008; 62: 423-5. 